Recibido: 8/10/2015

Aprobado versión definitiva: 15/11/2015

\title{
Marcos interpretativos de políticas públicas. el enfoque preventivo de la seguridad en el discurso político de candidatos a presidentes municipales de Guadalajara y Zapopan, Jalisco, México, en las elecciones de 2015
}

\author{
JANNY AMAYA TRUJILLO¹/ RADAMANTO PORTILLA TINAJERO²
}

\section{RESUMEN}

En este trabajo analizamos los marcos interpretativos desde los que se construyó el tema de la seguridad en el discurso político de los candidatos a las presidencias municipales de Zapopan y Guadalajara, durante las elecciones locales de 2015 en Jalisco -México. Asumimos como perspectiva heurística la teoría del framing. A partir de ella analizamos los supuestos desde los que se construye la seguridad como asunto de política pública. El resultado del análisis muestra el predominio del enfoque preventivo de la seguridad, en torno al que se estructuran siete marcos específicos: desigualdad, co-responsabilidad, criminalización de los jóvenes, corrupción e incapacidad policial, ocupación del espacio público, hipervigilancia y desvinculación.

Palabras clave: Discurso Político; Marcos interpretativos; Teoría del Framing; Seguridad; Prevención; Políticas públicas.

\footnotetext{
1 Doctora en Ciencias Sociales de la Universidad de Guadalajara, México. Afiliada al Instituto de Gestión del Conocimiento y del Aprendizaje en Ambientes Virtuales, Sistema de Universidad Virtual de la Universidad de Guadalajara. Av. La Paz \# 2453, Col. Arcos Sur. Código postal 44140, Guadalajara, Jalisco, México. Correo electrónico: jannyamaya@gmail.com.

2 Doctor en Ciencias Sociales de la Universidad de Guadalajara, México. Afiliado a Centro Universitario de Ciencias Sociales y Humanidades de la Universidad de Guadalajara. Parres Arias \#150, Sede Los Belenes. Código postal 44140, Guadalajara, Jalisco, México. Correo electrónico: radamanto.portilla@gmail.com.
} 
FRAMES. PREVENTIVE APPROACH TO SECURITY ON POLITICAL DISCOURSE OF MAYORS CANDIDATES OF GUADALAJARA AND ZAPOPAN, JALISCO, MEXICO, IN THE 2015 ELECTIONS.

\section{ABSTRACT}

This paper analyzes the interpretive frameworks from which was built the issue of security in the political discourse of the candidates for mayors of Zapopan and Guadalajara, during the local elections in 2015 in Jalisco, Mexico . We assume as heuristic perspective framing theory. From it we analyze the cases from which the security is built as a matter of public policy. The result of the analysis shows the prevalence of the preventive approach to security, around which seven specific frameworks are structured : inequality, co- responsibility, criminalization of youth, police corruption and incapacity, occupation of public space, hypervigilance and disengagement .

Keywords: Political discourse, Policy Frames, Framing theory, Security, Prevention, Public policy.

\section{Introducción}

La seguridad constituyó un tema de atención fundamental en la agenda política de los candidatos a munícipes de la Zona Metropolitana de Guadalajara en las elecciones del presente año (2015). Desde distintas posturas, este tema fue ampliamente abordado y desarrollado por los candidatos quienes ofrecieron, como parte de sus promesas de campañas, interpretaciones y soluciones diversas (convergentes unas, contradictorias otras) acerca de las causas, las consecuencias y las posibles soluciones a los persistentes y sentidos problemas de inseguridad que enfrenta el municipio.

En este trabajo analizamos los marcos desde el que fue construido y enmarcado el tema de la seguridad en el discurso político de los candidatos a las alcaldías de los municipios de Zapopan y Guadalajara. Para ello, asumimos como marco teórico y conceptual la teoría del framing o enmarcado, y retomamos algunos de los presupuestos centrales de su aplicación al análisis de los marcos 
retóricos de las políticas públicas (policy frames). El presupuesto fundamental que anima este tipo de análisis es que el modo en que se construyen y enmarcan discursivamente los problemas sociales tiene implicaciones importantes, tanto en el tipo de soluciones políticas que se implementan para resolver dichos problemas como en la conformación de la opinión pública en torno a ellos.

El trabajo está estructurado en cuatro apartados fundamentales. En el primero, recuperamos algunos de los conceptos y planteamientos fundamentales de la teoría del framing, particularmente en el área de los estudios sobre comunicación. En el segundo, presentamos un breve estado de la cuestión en la investigación sobre framing y políticas públicas. En el tercer apartado exponemos el diseño metodológico de la investigación. Por último, describimos y discutimos los principales resultados del análisis.

\section{El análisis de los marcos en los estudios de comunicación}

Previo a la consolidación y proliferación de la teoría del framing en el campo de la comunicación, el análisis de los marcos (frames) como objeto de estudio lo encontramos en las disciplinas de la sociología y la psicología. Bateson (1985, 2006) acuña el término marco de referencia o contextual para dar cuenta de una clase o conjunto de mensajes que forman estructuras lógico-cognitivas, representadas en el lenguaje (juego, guerra, tarea, responsabilidad, paternidad, etc.) o implícitas en las acciones de los individuos, que dan sentido y permiten comprender los discursos. El sentido sociológico del marco lo aporta Goffman $(1986,1997)$ en sus estudios sobre las interacciones sociales. Este autor plantea que los individuos, antes de actuar, se hacen una idea de la situación recurriendo a conocimientos previos $\mathrm{u}$ otras experiencias adquiridas en interacciones similares. A partir de ellos, los individuos definen la situación y actúan de acuerdo con las orientaciones que el marco posibilita. Esto quiere decir que las acciones de las personas no responden estrictamente a los hechos objetivos, sino a las interpretaciones que se hacen de los mismos (Sádaba, 2001). 
El análisis de los marcos tuvo poco impacto en el campo de la comunicación durante las décadas de los años 70 y 80. Fue a partir de 1995 que los investigadores en comunicación se apropiaron de este objeto de estudio. ${ }^{3}$ Pero fue durante el presente siglo cuando el análisis de los marcos, encumbrado bajo el nombre de teoría del framing, constituyó el principal interés de los estudios de comunicación en masas (Bryant \& Miron, 2004).

Entman (1993) aporta una de las más importantes (y vigentes) definiciones del proceso de enmarcado (framing): se entiende como el proceso de "seleccionar algunos aspectos de una realidad percibida y hacerlos más relevantes en un texto comunicativo, de tal manera que promueva una definición particular del problema, una interpretación de las causas, una evaluación moral y/o una recomendación de tratamiento"4 (p. 52) para el tema o asunto descrito. El supuesto que subyace en la teoría del framing es que un tema, asunto o acontecimiento puede ser visto desde una variedad de perspectivas y, según el tipo de marco con el que se le construya, tendrá implicaciones en múltiples aspectos de la sociedad: valores, opiniones, conductas, sensaciones.

En síntesis, los marcos (frames) ${ }^{5}$ consisten en un conjunto de principios organizativos (Reese, 2001, 2007) o ideas organizadoras centrales (Tankard, 2008) que están presentes en los discursos y la cultura. Además, se construyen y comparten socialmente, persisten en el tiempo y operan al nivel de los significados, símbolos e interpretaciones que estructuran el mundo social. Estos marcos se presentan como modelos o esquemas que organizan el significado y las interpretaciones que estructuran el mundo (véase Chong \& Druckman, 2007; Reese, 2007; Castells, 2010).

Actualmente, el análisis de los marcos es objeto de interés para distintas disciplinas científicas, aunque la comunicación ha encontrado en él un fructífero

\footnotetext{
3 Al respecto puede consultarse el trabajo de Bryant y Miron (2004).

4Cursivas en el original.

5Los términos de marco o encuadre se utilizan como sinónimos en la literatura hispanohablante para referirse a los frames. Sádaba (2007) hace una excelente reflexión en torno a la pertinencia de traducir el término frame como encuadre y no como marco.
} 
campo de producción empírica y analítica. Dentro de la ciencia política, otra área de aplicación de este enfoque se ha estructurado en torno al interés del análisis de la construcción discursiva de las políticas públicas. A esta área, denominada policy frames, le dedicamos el siguiente apartado.

\section{Policy frames: la investigación sobre framing y políticas públicas}

La adopción en el ámbito de las ciencias políticas del enfoque del framing se vincula, en gran medida, al trabajo de Martin Rein y Donald Schön (2002). Para Schön (Como se cita en Hulst \& Yanow, 2014) el enmarcado de políticas públicas puede ser comprendido como un proceso de producción de sentido: "Con el fin de convertir una situación problemática en un problema, los actores (políticos) deben hacer un determinado tipo de trabajo: deben dar sentido a una situación incierta" p. 6). El framing permite a los actores comprender una situación como de un cierto tipo (categorizarla) y comenzar a imaginar lo que podría o debería pasar a continuación. Ello a la luz de las nociones previas relativas a las formas en que este tipo de problemas pueden y deben ser manejados. Estos autores distinguen dos tipos de marcos en el análisis de asuntos de política pública: los marcos retóricos, activados en el debate en torno a problemas de política pública; y los

marcos de acción, que informan y dan sentido a los programas, leyes y regulaciones (Rein y Schön 1994 en Hulst \& Yanow, 2014, pp. 5-7).

En general, los estudios sobre policy frames o marcos interpretativos de políticas públicas comparten un supuesto básico: el modo en que se construyen y enmarcan discursivamente los problemas sociales tiene implicaciones importantes, tanto en el tipo de soluciones políticas que se implementan para resolver dichos problemas, como en la conformación de la opinión pública en torno a ellos. Desde este supuesto, se han estructurado tres vertientes de investigación predominantes: 1) estudios sobre el enmarcado de problemas sociales en discursos públicos y debates políticos y mediáticos; 2) estudios que analizan los marcos que estructuran y dan sentido a los instrumentos de política pública; y 3) estudios que analizan las relaciones entre el enmarcado de problemas sociales y 
la formulación de políticas públicas.

En el primero de estos ejes se ubica, por ejemplo, el trabajo de Lima \& Siegel (1999) quienes analizaron la cobertura informativa en torno a las políticas de regulación del tabaco en la prensa norteamericana entre 1997 y 1998; contexto en el que la formulación de estas políticas estaba siendo sometida a debate en el congreso estadounidense. Otros trabajos de este tipo se han centrado en el análisis de los marcos retóricos predominantes en debates políticos y parlamentarios. Magnani (2011) ha analizado la estructura y las transformaciones de los marcos dominantes en el debate político oficial en torno al control de la inmigración en Italia, durante las tres últimas décadas. En esta misma línea, Epstein, Roth y Baumer (2014) se han centrado en el análisis del lenguaje utilizado en el Foro de Gobernanza e Internet (2006-2011) para identificar los marcos utilizados por diferentes grupos en la retórica política acerca de la privacidad en línea. Por su parte, Ferraiolo (2014) ha examinado la dimensión moral y su extensión en el debate en torno a la legalización de la marihuana en los EE.UU, a partir de un análisis de los encuadres en un corpus conformado por declaraciones públicas, debates legislativos y documentos de campaña preparadas por los opositores a la despenalización como parte de las campañas legislativas. Shim, Park y Wilding (2015) realizaron un análisis de redes semánticas para investigar los marcos de política de energía nuclear en EE.UU., Reino Unido, Alemania, Francia, Japón y Corea del Sur.

En el segundo eje, centrado en el análisis de los marcos normativos de políticas públicas, se reconoce, por ejemplo, el trabajo de Luján Cunial (2014), que aborda el enmarcado del uso de drogas ilegales en el corpus normativo de políticas públicas en torno a este asunto, vigentes en Argentina durante el año 2014.

En el tercer eje o tendencia de investigación enfocada en el análisis de las relaciones entre el enmarcado de problemas sociales y la formulación de políticas públicas, o entre marcos retóricos y marcos normativos, se inscriben trabajos como los de Bustelo y Lombardo (2006). Estos autoresrealizaron una comparación 
de los marcos interpretativos de las políticas de igualdad de género entre la Unión Europea y seis de sus países miembros. En esta misma tendencia, autores como Toshkov y Wieldraaijer (2013) describen la transformación de los marcos de la política pública en torno a las drogas blandas en Holanda, durante el periodo comprendido entre 1990 y 2012. Otros estudios, como los de Rose y Baumgartner (2013) analizan la transformación en el debate público en torno a la pobreza en Estados Unidos a lo largo de alrededor de 50 años. También muestran cómo, en la medida en que ha cambiado el enmarcado público de este asunto, se han transformado también las políticas públicas en torno a la pobreza y ha disminuido la inversión gubernamental en este rubro.

En general, puede observarse un interés común en estas distintas vertientes de estudio: intención de describir y explicar por qué y cómo varía la definición y regulación de asuntos públicos, y la atención a la dimensión discursiva y simbólica de éstos. Así, se han desarrollado tanto análisis diacrónicos, que estudian las transformaciones de la política pública sobre un asunto particular en un mismo contexto a lo largo del tiempo, como análisis sincrónicos que abordan casos específicos o establecen comparaciones entre el enmarcado de un asunto común en distintos contextos nacionales. Aunque abordan sectores disímiles, todos estos trabajos comparten también el interés por la identificación o inferencia de marcos específicos, que dan sentido a la acción pública con respecto a los asuntos considerados como socialmente relevantes y problemáticos.

\section{Diseño Metodológico}

Para el análisis de los frames de la seguridad en el discurso político de los candidatos a las alcaldías de Zapopan y Guadalajara, asumimos un diseño metodológico cualitativo, basado en la técnica del análisis discursivo. Para ello, elaboramos un modelo de análisis basado en la propuesta de Entman (1993). A partir de la definición ofrecida por este autor, se delimitan cuatro funciones del marco: 1) Definición del problema; 2) Diagnóstico o identificación de las causas; 3) Valoración; y 4) Alternativas de solución. Estas cuatro funciones fueron asumidas como dimensiones básicas de análisis. En relación con cada una de ellas, fueron 
planteadas preguntas específicas a modo de indicadores que permitieran su operacionalización en relación con el tema de la seguridad (Véase Cuadro 1).

Otra de las dimensiones planteadas para el análisis fue la de Dispositivos de construcción del marco. En este sentido, identificamos palabras clave, frases hechas y estereotipos (Entman, 1993), que se utilizan en la definición y descripción del problema; así como las metáforas asociadas a la seguridad en el discurso de los candidatos. Además, procuramos detectar las claves periféricas que operan como recursos de simplificación del marco y que debido a su resonancia cultural funcionan incluso en la ausencia de una evaluación exhaustiva de los argumentos del marco (Muñiz, 2011).

La última fase del procedimiento de análisis consistió en la inferencia de los micro-frames y macro-frames. Reese $(2001 ; 2008)$ propone distinguir entre micro y macro-frames en dependencia del grado de organización que ellos llegan a alcanzar. Un mismo macro-frame puede estructurar así varios micro-frames subordinados, que son mucho más locales o específicos, en tanto se vinculan con contenidos particulares del frame general o macro-frame.

El corpus de análisis estuvo constituido por dos grandes grupos de documentos: entrevistas realizadas a los candidatos en noticieros de televisión locales $^{6}$ y debates televisados organizados por el Instituto Electoral y de Participación Ciudadana del Estado de Jalisco (IEPCJ), por la Universidad de Guadalajara y por el colectivo ciudadano Indagantes, durante la campaña electoral desarrollada entre los meses de abril y junio de 2015. El criterio fundamental para la selección del corpus fue que abordaran, aunque fuese de modo muy breve, el asunto de la inseguridad.

De las entrevistas, fueron seleccionadas como unidades de análisis las respuestas ofrecidas por los candidatos a interrogantes en torno al tema de la seguridad. De los debates, se seleccionaron las intervenciones y réplicas de los

6Es importante destacar que el acceso a estas entrevistas fue posible gracias a la participación de una de las autoras de esta ponencia en el Monitoreo de Radio y Televisión desarrollado por el Departamento de Estudios de la Comunicación Social de la Universidad de Guadalajara durante la campaña estudiada. 
candidatos sobre este tema específico. Se recuperaron un total de 13 entrevistas a candidatos de Guadalajara y 12 a candidatos de Zapopan realizadas en los noticieros Meganoticias, Señal Informativa, Hechos Meridiano y Hechos Noche. Además las intervenciones en los debates organizados por el IEPCJ, el Colectivo Indagantes y el Debate Académico de la Universidad de Guadalajara.

Para llevar a cabo el procesamiento de la información utilizamos el software de análisis cualitativo AtlasTi 6 que facilitó la identificación de los fragmentos discursivos, la organización y codificación de la información recolectada (Ver cuadro . 


\section{Cuadro 1. Modelo de análisis}

\begin{tabular}{|c|c|c|c|c|}
\hline $\begin{array}{l}\text { FUNCIONES } \\
\text { MARCO }\end{array}$ & $\begin{array}{l}\text { PREGUNTAS ESPECÍFICAS PARA EL } \\
\text { ANÁLISIS DE LAS FUNCIONES DEL } \\
\text { MARCO }\end{array}$ & $\begin{array}{l}\text { DISPOSITIVOS DE } \\
\text { CONSTRUCCIÓN DEL } \\
\text { MARCO }\end{array}$ & $\begin{array}{l}\text { FRAME } \\
\text { (específico o } \\
\text { subordinado) }\end{array}$ & META-FRAME \\
\hline \multirow[t]{4}{*}{$\begin{array}{l}\text { Definición } \\
\text { problema }\end{array}$} & $\begin{array}{l}\text { ¿Qué se entiende por seguridad? } \\
\text { ¿Qué "problemas" o situaciones se } \\
\text { asocian con la seguridad? } \\
\text { ¿Quiénes son los afectados por la } \\
\text { inseguridad? } \\
\text { ¿Cómo se define el problema? }\end{array}$ & \multirow{14}{*}{$\begin{array}{l}\text { Palabras clave } \\
\text { (Keywords) } \\
\text { Frases hechas } \\
\text { Estereotipos } \\
\text { Metáforas: } \\
\text { (asociaciones, } \\
\text { analogías, diferencias) }\end{array}$} & \multirow{14}{*}{$\begin{array}{l}\text { (Marcos } \\
\text { interpretativo } \\
\text { s específicos } \\
\text { mediante los } \\
\text { cuales se } \\
\text { enmarca } \\
\text { temas, } \\
\text { acontecimie } \\
\text { ntos, o } \\
\text { situaciones } \\
\text { particulares) }\end{array}$} & \multirow[t]{14}{*}{$\begin{array}{l}\text { (Marco interpretativo general } \\
\text { en torno al cual se organizan } \\
\text { simbólicamente los micro- } \\
\text { frames) }\end{array}$} \\
\hline & ¿Por qué se considera como problema? & & & \\
\hline & $\begin{array}{l}\text { ¿Cuáles son los efectos o implicaciones } \\
\text { que se le atribuyen? }\end{array}$ & & & \\
\hline & $\begin{array}{l}\text { ¿Qué agentes, sujetos o grupos sociales } \\
\text { se asocian al problema? }\end{array}$ & & & \\
\hline \multirow{3}{*}{$\begin{array}{l}\text { Diagnóstico } \\
\text { identificación de las } \\
\text { causas }\end{array}$} & $\begin{array}{l}\text { ¿Cómo se diagnóstica el problemas? } \\
\text { ¿Cuáles son las causas que lo originan? }\end{array}$ & & & \\
\hline & $\begin{array}{l}\text { ¿Quiénes son los actores a los que se } \\
\text { les atribuye el problema? }\end{array}$ & & & \\
\hline & $\begin{array}{l}\text { ¿Las causas son justificadas con } \\
\text { argumentos científicos, experienciales, } \\
\text { morales o no se justifican? }\end{array}$ & & & \\
\hline \multirow{3}{*}{$\begin{array}{l}\text { Evaluación } \\
\text { Valoración } \\
\text { problema }\end{array}$} & $\begin{array}{l}\text { ¿Cuál es la valoración del problema? } \\
\text { (positiva, negativa o neutral) }\end{array}$ & & & \\
\hline & Hay apelación a las emociones & & & \\
\hline & $\begin{array}{l}\text { ¿Qué implicaciones morales se } \\
\text { atribuyen al problema? }\end{array}$ & & & \\
\hline \multirow[t]{4}{*}{$\begin{array}{l}\text { Alternativas } \\
\text { solución }\end{array}$} & $\begin{array}{l}\text { ¿Qué soluciones se proponen al } \\
\text { problema? }\end{array}$ & & & \\
\hline & $\begin{array}{l}\text { ¿Las soluciones presentadas han sido } \\
\text { probadas o tienen referente en otros } \\
\text { contextos? }\end{array}$ & & & \\
\hline & $\begin{array}{l}\text { ¿Qué predicciones se generan sobre el } \\
\text { problema? }\end{array}$ & & & \\
\hline & $\begin{array}{l}\text { ¿De qué tipo son las soluciones } \\
\text { ofrecidas: preventivas, reactivas, } \\
\text { punitivas, normativas (de control), de } \\
\text { vigilancia, etc.? }\end{array}$ & & & \\
\hline
\end{tabular}

Fuente: Elaboración propia a partir del modelo propuesto por R. Entman (1993). 


\section{Interpretación de los hallazgos: el predominio del marco preventivo}

En el análisis de los extractos discursivos obtenidos de los debates y las entrevistas se identificó el macro-frame de la prevención como idea central organizadora de los argumentos en torno a la seguridad y la construcción de las políticas públicas. En torno al marco preventivo se organizan un conjunto de siete micro-frames que se distinguen y diferencian por los supuestos que subyacen en el tipo de alternativas de solución ofrecidas o el énfasis en determinadas causalidades del problema en cuestión. Estos micro-frames son transversales, es decir, no se localizan o se identifican plenamente en los discursos de candidatos específicos, sino que atraviesan la retórica de todos los candidatos y son empleados indistintamente por ellos, aunque es posible distinguir cierto predominio según el actor que se trate ${ }^{7}$.

De acuerdo con estos hallazgos sostenemos que el discurso político de los candidatos a las presidencias municipales de Guadalajara y Zapopan, durante la elección intermedia de junio del 2015, estuvo enmarcado predominantemente por el enfoque preventivo de la seguridad. Con ciertos matices que serán expuestos más adelante, todos los candidatos estructuraron sus discursos y dieron sentido a sus propuestas bajo la idea del enfoque preventivo. La predominancia del enfoque preventivo en las propuestas de política de los candidatos era de esperarse dada la crítica global respecto a la ineficacia de los modelos policiacos generados bajo la concepción de la seguridad pública. En ese sentido, la adopción del macroframe preventivo -que emerge del concepto de seguridad ciudadana- como marco orientador en la construcción de políticas públicas responde a la tendencia, nacional ${ }^{8}$ y mundial, por privilegiar la atención de los factores que inciden en la delincuencia y la violencia.

7 En los siguientes apartados se presentan esos matices en el uso de los micro-frames según los discursos de los candidatos.

8 A final de la administración de Felipe Calderón Hinojosa (2006-2012) se promulgó la Ley General para la Prevención de la Violencia y la Delincuencia, misma que establece: "[las políticas públicas deben estar] orientadas a reducir los factores de riesgo que favorezcan la generación de la violencia y delincuencia, así como combatir las distintas causas y factores que la generan" (Art. 2, 
Debido al tipo de discursos analizados, la función del marco que se identificó con mayor precisión fue las alternativas de solución. La función de identificación de las causas se presentó de dos maneras en los discursos: explícitamente, cuando presentaban argumentos que revelaban la teoría causal del problema de la inseguridad, en algunos casos con evidencia empírica o documental; e implícitamente, de acuerdo con el tipo de propuestas presentadas, que mediante un proceso de inferencia lógica permiten conocer cuáles son las causas de la inseguridad que pretenden atender las propuestas.

La identificación de las funciones del marco definición del problema y evaluación del problema en los extractos discursivos presentó ciertas complicaciones metodológicas. La mayoría de los discursos de los candidatos carece de una definición, concepción o interpretación explícita de la seguridad. Sin embargo, ésta subyace en las alternativas o propuestas de solución del problema. Por lo tanto, fue necesario un procedimiento de inferencia de los supuestos que dan sentido a las propuestas presentadas. Dichos supuestos se presentan aquí como las concepciones o definiciones del problema de la seguridad. Igualmente para la función evaluación del problema, cuya intención es identificar las valoraciones morales, ideológicas o emocionales contenidas en los discursos sobre la seguridad. Cuando fue posible se infirieron dichas valoraciones a partir de los dispositivos de construcción del marco.

Ley General para la Prevención de la Violencia y la Delincuencia, disponible en http://dof.gob.mx/nota_detalle.php?codigo=5230810\&fecha=24/01/2012) 
Cuadro 2. Marcos interpretativos de la inseguridad en los discursos de los candidatos a las presidencias municipales de Guadalajara y Zapopan

\begin{tabular}{|l|l|}
\hline \multirow{4}{*}{ MACRO-FRAME } & \multirow{2}{*}{ MICRO-FRAME } \\
\hline \multirow{4}{*}{ El enfoque preventivo de la seguridad } & De la desvinculación \\
\cline { 2 - 2 } & De la co-responsabilidad \\
\cline { 2 - 2 } & De la desigualdad estructural \\
\cline { 2 - 2 } & De la criminalización de la juventud \\
\cline { 2 - 2 } & Del espacio público \\
\cline { 2 - 2 } & Del control y la hiper-vigilancia \\
\cline { 2 - 2 } & De la corrupción e incapacidad policiaca \\
\hline
\end{tabular}

Fuente: Elaboración propia.

a. La prevención como macro-frame de la inseguridad: atender a las causas, no a los efectos.

El enfoque preventivo se constituyó como el principal recurso retórico y de enmarcado de los discursos de los candidatos a las presidencias municipales de Guadalajara y Zapopan. El supuesto que subyace en este macro-frame es la idea de que las políticas públicas deben orientarse a la atención de los factores, causas o condiciones que generan o posibilitan la inseguridad. Las diferencias radicaron en el diagnóstico que hicieron de la inseguridad y, por tanto, en los factores, causas o condiciones que identificaron como responsables del problema. Dichas diferencias responden a procesos de enmarcamiento particulares -formas de seleccionar, enfatizar y priorizar algunos aspectos del problema- que pueden coexistir, transponerse y compartir aspectos sin contradecir o debilitar el sentido del macro-frame y que serán explicados en siguientes apartados de este trabajo. 
Marcos Interpretativos de Políticas Públicas. el Enfoque Preventivo de la Seguridad...

Los siguientes extractos del discurso ejemplifican el enfoque preventivo del macro-frame:

Atendiendo las causas, no los efectos del problema. El planteamiento de fondo que nosotros estamos haciendo es que nos concentremos en atender los factores que están incidiendo en el incremento de la delincuencia en la ciudad... Nosotros lo que vamos a cambiar es el modelo para orientarlo a la prevención... (Enrique Alfaro, Candidato de Movimiento Ciudadano, Guadalajara, en entrevista Meganoticias).

Enfocar las políticas principalmente a la prevención. La solución está en la prevención, por eso la necesidad de una policía comunitaria, una visión metropolitana. (Alfonso Petersen, Candidato del Partido Acción Nacional, Guadalajara, en Debate Indagantes)

El macro-frame de la prevención establece como aspectos de la inseguridad delitos relacionados con el robo, en cualquiera de sus modalidades: a personas, a inmuebles, a vehículos. Los pocos diagnósticos presentados por los candidatos durante las entrevistas o los debates versan en torno a estos delitos. Algunos candidatos incluyeron en sus discursos menciones relacionadas al crimen organizado $^{9}$, pero solo sirvieron para ilustrar el fracaso de las autoridades en el problema de la inseguridad y el grado de violencia que se presenta en la entidad.

En cierta medida, la acotación del problema de inseguridad a delitos como el robo es coherente con las facultades de las policías municipales. Sin embargo, en el discurso de los candidatos se ocultó -u omitió- otros delitos del fuero común en los que las autoridades municipales tienen algunas atribuciones (de prevención e investigación, por ejemplo). Tal es el caso de los homicidios, el secuestro, la violación o las agresiones físicas, entre otros:

Lo que más aqueja a los ciudadanos es el robo común, el asalto a menores. (Jorge Real Ramírez, candidato del Partido Encuentro Social, Zapopan, en Debate IEPCJ)

La meta será bajar en $25 \%$ el robo a mano armada, asaltos en vía pública y robo a casa habitación. (Enrique Velázquez, candidato del PRD, Zapopan, en Debate IEPCJ)

9 Específicamente se hizo referencia a los "narcobloqueos" que se presentaron en todo el estado de Jalisco el 1 de mayo de 2015. 
A continuación se expondrán los siete micro-frames identificados, en torno a los que se organizan tanto las causas como las alternativas de solución propuestas por los distintos candidatos. Reiteramos, no obstante, que ellos deben ser comprendidos en estrecha relación con el macro-frame de la prevención, del cual son sólo variaciones o manifestaciones puntuales, localizadas en torno a aspectos específicos del problema.

A. La inseguridad asociada a la desvinculación: de la policía cercana a la reconstrucción del tejido social

Desde este micro-frame, la inseguridad se construye como un problema asociado a la ruptura o la debilidad de vínculos a nivel institucional y social. El supuesto de fondo es que la calidad o la disfuncionalidad de las relaciones (de integración, de cercanía, o de coordinación) entre sujetos y comunidades, entre instituciones y ciudadanía o entre instituciones constituyen una de las causas de la inseguridad. Se reconocen tres tipos o niveles fundamentales de ruptura 0 carencia de vínculos identificados como causas directas o indirectas de la inseguridad.

En primer lugar, se construye a la inseguridad como un asunto asociado a la desvinculación entre instituciones y ciudadanía. Concretamente, desde esta perspectiva, la lejanía y el desconocimiento mutuo entre la institución policíaca y los ciudadanos es una de las causas de la inseguridad:

Yo lo que digo es que los policías no pueden ser extraños, dando vueltas todo el día en las colonias, debe ser gente que conozcamos. (...). Necesitamos recuperar una policía cercana, reabrir los módulos, hacer que los policías se acerquen con los jóvenes, que tengan contacto con los ciudadanos (Ricardo Villanueva, Candidato del PRI, Guadalajara, en entrevista Meganoticias).

Los policías serán efectivos cuando recuperen la confianza de los ciudadanos, lo que se logra con una verdadera vinculación. (Enrique Velázquez, Candidato del PRD, Zapopan, en debate IEPCJ)

Una metáfora recurrente en la construcción de este micro-frame es la del contacto y la cercanía como base para la construcción de la confianza. Según esta lógica si policías y ciudadanos se reconocen mutuamente, si están en 
contacto "directo", si establecen incluso relaciones de índole personal, es posible entonces confiar en ellos, y esta confianza mutua es una condición deseable para la resolución del problema de la inseguridad.

Un segundo nivel en el que se localiza o identifica esta carencia de vínculos es al nivel de las instituciones, es decir, la disfuncionalidad de los vínculos o conexiones entre instituciones distintas es otra de las causas identificadas de la inseguridad. La falta de coordinación entre diferentes instituciones del mismo nivel (como los cuerpos policiacos metropolitanos) o entre instituciones de niveles diferentes (como el gobierno estatal y federal), son causas de la inseguridad:

La ciudad no puede seguir teniendo 11 mandos distintos, con 11 estrategias diferentes en la misma ciudad, necesitamos una sola visión de seguridad para la Zona Metropolitana de Guadalajara. (Enrique Alfaro, Candidato MC, Guadalajara, en entrevista Meganoticias)

Tiene que haber absoluta coordinación, no podemos desconocer que estamos en una metrópoli donde los distintos municipios tenemos problemas similares, que requieren que trabajemos soluciones en conjunto. (Salvador Rizo, Candidato PRI, Zapopan, en entrevista Meganoticias)

Las metáforas más recurrentes en este sentido son las de la coordinación y la integración. La falta de coordinación, particularmente entre las instituciones policiacas, es una de las causas de la incapacidad del gobierno para solucionar el problema de la inseguridad. La efectividad en la solución a este problema sólo puede conseguirse a partir de una acción coordinada, o incluso, integrada a nivel institucional.

Por último, este micro-frame se localiza, además, en discursos que reconocen en el debilitamiento de los vínculos sociales otra de las causas indirectas de la inseguridad. Los supuestos de fondo son los siguientes: el retraimiento del individuo hacia la esfera privada ha afectado negativamente la construcción de nexos sociales y comunitarios más amplios. Ello se asocia a la pérdida de valores e intereses comunitarios y a un declive de la participación en la vida pública. Este debilitamiento de los vínculos sociales ha traído como 
consecuencia una suerte de pérdida de presencia ciudadana, una pérdida de valores, que abonan indirectamente al incremento de la inseguridad:

Vamos a trabajar en la re-construcción del tejido social, que es la única forma en la que vamos a recuperar la paz. (Enrique Alfaro, Candidato MC, Guadalajara, en entrevista Meganoticias)

La desconfianza e indiferencia hacia el entorno social provoca inseguridad. (Jorge Leal, Candidato PES, en Debate Zapopan)

En materia de acciones o alternativas de solución, esta variante del microframe informa y da sentido a propuestas que buscan precisamente el fortalecimiento de relaciones sociales, o la creación de comunidades, como la organización de orquestas sinfónicas en las colonias con mayores índices delictivos (Marco Tulio Rosas, Candidato Morena, Zapopan); la creación de espacios deportivos (Enrique Alfaro, Candidato MC, Guadalajara; Isaac Pérez Gómez, Candidato PT, Zapopan); o la recuperación de espacios públicos que faciliten la convivencia familiar y comunitaria (Salvador Rizo, Candidato del PRI, Zapopan; Pablo Lemus, Candidato MC, Zapopan). Este micro-frame se asocia muy estrechamente al de la Recuperación del espacio público, que desarrollamos en otro apartado de este trabajo.

B. La inseguridad asociada a la co-responsabilidad: de la consulta pública al ciudadano centinela

En este micro-frame, el problema de la inseguridad se plantea como un asunto que no puede resolverse única y exclusivamente desde la acción pública. El supuesto que subyace en el micro-frame de la co-responsabilidad es que la consecución de resultados efectivos y contundentes contra la inseguridad solamente podrá conseguirse mediante un trabajo colectivo que implique la participación de la sociedad y los órganos de gobierno. Con este micro-frame, los candidatos atribuyen un grado de responsabilidad a la sociedad pero nunca aclaran en qué sentido o de qué tipo.

La co-responsabilidad en el problema de la seguridad no significa la transferencia de las funciones del Estado a la sociedad, como son las del ejercicio de la fuerza, la impartición de justicia, la aplicación de sanciones, sino un tipo de 
colaboración entre la sociedad y el gobierno, principalmente con las instituciones encargadas de la seguridad. Dicha participación ciudadana parece quedar circunscrita a tres modalidades de actuación: de consulta o validación, de vigilancia de las policías y de denuncia.

La participación ciudadana en la modalidad de consulta se expresa en la conformación de consejos ciudadanos de seguridad pública u otras formas similares de organización social como los consejos consultivos, los comités de prevención, etc., Esta propuesta es una de las más recurridas por los candidatos, como se evidencia en los discursos de Salvador Rizo, candidato del PRI, Zapopan; Alfonso Petersen, candidato del PAN, Guadalajara y Guillermo Martínez Mora, candidato del PAN, Zapopan. Aparentemente, mediante estos organismos los ciudadanos serán co-responsables o co-partícipes de las políticas en materia de seguridad:

Estamos platicando con las personas y hemos visto que sería muy importante que los vecinos, las vecinas tomen parte de la toma de decisiones en lo que respecta al tema de seguridad... con la participación de los locatarios, de los vecinos y las decisiones que se tomen ahí van a ser vinculatorias, van a compartirse las responsabilidades entre la autoridad y la ciudadanía... (Marco Tulio Flores, Candidato de Morena, en entrevista Señal Informativa)

No se ha puesto en marcha el tema de los comités de policía vecinal, yo creo que un muy importante echar a andar un programa de policía vecinal. Donde nosotros como sociedad seamos los primeros responsables de la seguridad. (Luis Roberto Preciado, Candidato del PH, Zapopan, en entrevista Señal Informativa)

La otra modalidad de actuación en materia de participación ciudadana que se presenta en este micro-frame es aquella que atribuye la responsabilidad de vigilante o supervisor a la sociedad respecto de la actuación policial. En este sentido, los ciudadanos tienen la responsabilidad de vigilar y denunciar a los elementos policiacos que no cumplan sus funciones:

Un buzón anónimo, un buzón ciudadano en las colonias para que me reporten si el policía no está respondiendo a sus demandas, pero sobre todo para fortalecer la confianza en la policía que está muy abajo, muy desmejorada... (Beatriz García de la Cadena, Candidata del PT, Guadalajara, en entrevista Meganoticias) 
Por último, el micro-frame de co-responsabilidad admite también la denuncia ciudadana como otra modalidad de participación de la sociedad. Esto presupone al menos dos formas de denuncia: la directa o presencial, que consiste en que los ciudadanos víctimas de algún delito acudan a las autoridades; y la anónima en la que los ciudadanos operan como centinelas, celadores o vigías de su entorno. La denuncia anónima se presenta, por tanto, como un dispositivo de atribución y transferencia de responsabilidad que solamente funciona bajo el supuesto de que hay inseguridad o comisión de delitos porque los ciudadanos no denuncian -no señalan, no acusan:

Una coordinación eficiente de donde están las patrullas y qué están haciendo, sus rondines el pase a empresas para que les sellen que sí pasaron por la vigilancia, etc. Una estrategia de denuncia ciudadana... (Leonardo García Camarena, entrevista Meganoticias)

Las metáforas que subyacen en el micro-frame de co-responsabilidad son la seguridad comienza en casa y la del ciudadano centinela. De particular importancia parece estar operando la metáfora del centinela en este micro-frame debido a que la participación ciudadana adquiere mayor fuerza y sentido social cuando se trata de vigilar, denunciar y exigir. En ese sentido parecen operar, incluso, los consejos ciudadanos y las distintas formas de organización, aparentemente incluyentes, de la ciudadanía en torno al problema de la inseguridad.

El micro-frame de co-responsabilidad admite, principalmente, propuestas del tipo Consejos Ciudadanos de Seguridad Pública (Alfonso Petersen, candidato del PAN, Guadalajara; Guillermo Martínez Mora, candidato del PAN, Zapopan; Salvador Rizo, candidato del PRI, Guadalajara) o Comités Vecinales para la Prevención del Delito (Guillermo Martínez Mora, candidato del PAN, Zapopan; Beatriz García de la Cadena, candidata del PT, Guadalajara), entre otros. También abundan las propuestas que se orientan en incrementar la denuncia ciudadana con la modernización de los mecanismos de denuncia y la implementación de nuevos filtros para garantizar la protección y el anonimato de las víctimas y testigos: Buzón Ciudadano (Beatriz García de la Cadena, 
Candidata del PT, Guadalajara) o estrategia de denuncia ciudadana (Leonardo García Camarena, Candidato del Partido Humanista, Guadalajara).

C. La inseguridad asociada a la desigualdad: el problema de fondo

Desde este micro-frame, la desigualdad- entendida en términos amplios y abstractos como desigualdad económica, desigualdad de oportunidades, desigualdad en el acceso a la educación o desigualdad en la distribución de las fuentes de empleo- se construye como causa última de la inseguridad. El supuesto de fondo es que los agentes causales de la inseguridad, es decir, los delincuentes, según la definición predominante del problema, han carecido o carecen de otras alternativas de vida, que no han tenido acceso a la educación, al trabajo digno o a fuentes de ingresos económicos legales y suficientes. Delinquir es su única opción, o al menos, la más viable, y la desigualdad social- en sus diversas variantes o manifestaciones- es la causa última de esta carencia de alternativas. A continuación se presentan algunos ejemplos que evidencian lo antes planteado:

El problema de fondo está en la necesidad urgente de crear empleos para los jóvenes que se van a la delincuencia y la drogadicción. (Guillermo Martínez mora, Candidato del PAN, Zapopan, en debate Zapopan)

La mejor política social que existe en contra de la inseguridad es el empleo. (Pablo Lemus, Candidato MC, Zapopan, en entrevista Meganoticias)

El Estado debe estar garantizándoles su seguridad a partir de equilibrar entre los que más tienen como los que menos tienen. (Enrique Velázquez, Candidato del PRD, en entrevista Señal Informativa)

El discurso de los candidatos aborda distintas aristas o manifestaciones de la desigualdad; sin embargo, este micro-frame se concentra prioritariamente en torno a dos ejes fundamentales: la desigualdad en el acceso a la educación y la desigualdad en el acceso al empleo. El primero se presupone que elevando el nivel educativo se podrá insertar socialmente a los jóvenes y otorgarles indirectamente el acceso a otras alternativas de vida. En cuanto al otro - se asume que creando nuevas fuentes de empleo pueden garantizarse otras formas de vida distintas de la delincuencia o la ilegalidad. 
En ambos casos, se asume que son los jóvenes los agentes causales de la inseguridad. Por lo tanto, sólo a partir de políticas sociales redistributivas dirigidas, especialmente, a este sector de la población puede solucionarse de fondo el problema de la inseguridad. En todo caso, la desigualdad es asumida como causa estructural construida bajo la metáfora de la "causa de fondo". El micro-frame de la inseguridad asociada a la desigualdad se relaciona estrechamente con el de la criminalización y victimización de la juventud que será descrito más adelante.

Este micro-frame informa aquellas alternativas de solución que enfatizaron en políticas sociales, tales como la creación de escuelas de oficios (Villanueva, Candidato del PRI, Guadalajara). Además, en los programas de becas, como es el caso del programa Becarios sí, Sicarios no de Marco Tulio Rosas, Candidato de Morena, Zapopan. O el programa universal de becas a estudiantes de preparatoria propuestas por el candidado de MC Enrique Alfaro, por sólo citar algunos ejemplos.

D. La inseguridad como problema asociado a la juventud: ¿Cómo recuperamos a nuestros jóvenes?

Desde esta perspectiva, los jóvenes son identificados como agentes responsables (causantes) de la inseguridad. La delincuencia directamente asociada a la inseguridad, según la definición predominante del problema, es descrita como una actividad eminentemente juvenil. De este modo la juventud, la violencia y la delincuencia aparecen como términos estrechamente asociados en el discurso. La juventud es el único sector o grupo social al que se le atribuyen abiertamente responsabilidades sobre el problema, además de los policías. Sin embargo, este micro-frame aparece estrechamente relacionado con el micro-frame de la desigualdad. Sus supuestos causales son que los jóvenes no han tenido oportunidades educativas, laborales y de inserción social en general. Esta falta de oportunidades ha provocado que se involucren en adicciones, o que asuman la delincuencia como modo de vida. De este modo, se construye a la juventud en una doble condición: se criminaliza a los jóvenes al identificarlos como 
delincuentes; y se les victimiza al considerar que su comportamiento criminal es consecuencia de una condición estructural mayor (la desigualdad), que los ha privado de otras alternativas:

¿Cómo recuperamos a nuestros jóvenes? (...) Si no vamos y le damos una oportunidad a esos jóvenes, con policías y a macanazos no vamos a resolver los problemas. (...) Vamos a crear el primer centro de oficios, y entonces, aquel joven que decida rehabilitarse, y entrar a este centro de oficios, (se le otorgará) una beca durante dos años de 2 mil pesos al mes para que aprenda un oficio. A partir de ahí podrá ese joven dedicarse a vivir de su trabajo. (....)Con jóvenes sin oportunidades en nuestros barrios siempre vamos a tener los problemas de violencia que estamos teniendo. (Ricardo Villanueva, Candidato del PRI, Guadalajara, en entrevista Meganoticias)

Un proyecto de educación y más y mejores empleos para que los jóvenes no se infiltren, no se vayan a las filas del crimen organizado. (Enrique Velázquez, Candidato del PRD, en entrevista Señal Informativa)

Los jóvenes son descritos como un grupo social "perdido", desde la metáfora de la recuperación, y como un grupo vulnerable necesitado de protección, a partir de la etiqueta de "jóvenes sin oportunidades" o de la metáfora del apoyo. Esta última descripción de la juventud como grupo vulnerable no sólo se presenta en relación directa con la delincuencia (que los jóvenes no se infiltren en las filas del crimen organizado), sino también en asociación con la adicción: los jóvenes son adictos que deben rehabilitarse o corren el riesgo de serlo si no se les dota de otras oportunidades educativas, laborales y de uso del tiempo libre. En consecuencia- al igual que en el caso del micro-frame de la desigualdad-, este marco da sentido a propuestas orientadas hacia la formulación de políticas sociales enfocadas en la juventud.

Un recurso relativamente recurrente en la construcción de este marco fue la apelación a frases como "a macanazos", o "con policías y a macanazos", de alta resonancia cultural a nivel local. Esta frase, retomada de una declaración realizada por el actual alcalde priísta de Zapopan, Héctor Robles, para hacer alusión a sus políticas de enfrentamiento a la delincuencia juvenil, y que en su momento recibiera amplias críticas por parte de académicos y ciudadanos, operó como un recurso de simplificación del marco que sintetizaba un enfoque preventivo, 
opuesto a la reacción y la violencia policiaca como alternativas de solución ante la inseguridad.

E. La inseguridad asociada al espacio público: la delincuencia está ahí afuera

Este micro-frame establece una fuerte asociación entre la inseguridad y el uso de espacios públicos. Predominan, en este sentido, dos supuestos complementarios. Por una parte, se asume que el espacio público- calles, parques, unidades deportivas- ha sido abandonado y descuidado - no está iluminado, no es agradable, no es habitable-. El espacio abandonado es el espacio de la delincuencia:

Los zapopanos no visitan espacios públicos porque no están iluminados, porque están descuidados, porque tienen grafitis, porque la delincuencia está ahí. (Jorge Leal, Candidato PES, Zapopan, en Debate Zapopan)

No hay alumbrado público, no hay parques, y si no hay alumbrado público y no hay parques, pues son nidos de delincuencia a donde los manda. (Jesús Burgos, Candidato Morena, Guadalajara, en entrevista Meganoticias GDL 08)

Esos jóvenes están en la calle, en la calle se encuentran las drogas, las drogas cuestan dinero, y para conseguir ese dinero se están robando las baterías de los autos en la ciudad... (Ricardo Villanueva, Candidato del PRI, Guadalajara, en entrevista Meganoticias)

Esta concepción del espacio público como el espacio de la inseguridad es complementado en el discurso de algunos de los candidatos con un segundo supuesto, el cual se expresa como alternativa de solución al problema: si los espacios públicos se dotan de sentido, si la ciudadanía ocupa las calles, si se apropia de ellas, entonces éstas dejarán de ser el espacio de la delincuencia para convertirse en espacios de construcción y fortalecimiento de vínculos sociales:

Estamos planteando, políticas para que los espacios públicos sean mejores y tengan mucho más contenido y tengan un uso más intensivo, para recuperar el sentido de barrio, sentido de pertenencia a tu comunidad. Son los temas en los que creemos podemos ir reconstruyendo el tejido social, y poco a poco en ir bajando los niveles de delincuencia. (Enrique Alfaro, Candidato MC, Guadalajara, en entrevista Meganoticias)

Le estamos diciendo a la gente que necesitamos recuperar la vida de las colonias, si recuperamos la vida de los barrios y las colonias en automático empieza a bajar la inseguridad. (Leonardo García Camarena, Candidato de PH, Guadalajara, en entrevista Meganoticias) 
Algunas metáforas utilizadas en esta manifestación o variante específica del micro-frame son la de la recuperación y el rescate: los espacios públicos se han perdido, ya no son nuestros, en ellos ya no habita la vida, y por lo tanto, es necesario rescatarlos, recuperarlos. Además, en este caso la idea de recuperación del espacio público se asocia fuertemente con la construcción de ciudadanía, con la reconstrucción del tejido social; es decir, con el micro-frame de la desvinculación que explicamos anteriormente.

F. La inseguridad asociada al control y la hipervigilancia: que Guadalajara de noche parezca de día

El supuesto básico de este micro-frame es que el control y la vigilancia extrema funcionan como inhibidores de la comisión de delitos. Se presupone entonces que la falta de control es una de las causas de la inseguridad y que un sistema complejo de vigilancia permanente es una de las alternativas de solución a este problema social. Así, paradójicamente, el control y la hipervigilancia se conciben como recursos o alternativas preventivas ante el problema de la inseguridad.

Yo estoy planteando la red de videovigilancia más grande del país, tener $10 \mathrm{mil}$ videocámaras (...) Con esa red de videovigilancia se ha logrado detectar e inhibir el delito en muchas partes. Con 10 mil creo que vamos a hacer grandes cosas. (Ricardo Villanueva, Candidato del PRI, Guadalajara, en entrevista Meganoticias)

Todas las lámparas encendidas, una ciudad bien iluminada. Quiero que Guadalajara de noche parezca de día. (Ricardo Villanueva, Candidato del PRI, Guadalajara, en Debate IEPCJ)

Una de las metáforas sistemáticas utilizadas en la construcción de este micro-frame es la de la oposición entre la luz y la oscuridad: se asume que los delitos se cometen en lo oscuro, allí donde no son visibles. La inseguridad y la percepción de la inseguridad se asocian con la oscuridad, por tanto la iluminación y la visibilización son concebidos como recursos para combatirlos.

Según este micro-frame, los potenciales delincuentes son también los policías, quienes son identificados también como uno de los grupos responsables de la inseguridad. Algunas de las medidas de control planteadas por los 
candidatos se enfocan en dotar de cámaras de vigilancia a todas las patrullas (Ricardo Villanueva, Candidato del PRI, Guadalajara); dotar a los policías de cámaras en el pecho (Delait Pérez, Candidato del PANAL, Zapopan). También manifiestan el interés de transformar las unidades de monitoreo y ponerles un GPS que facilite su seguimiento y localización (Jorge Leal, Candidato del PES, Zapopan). Incluso, plantean la implementación de un sistema de cuarteles donde pueda garantizarse un control sobre la vida privada de los policías (Guillermo Martínez Mora, Candidato del PAN, Zapopan).

G. La inseguridad asociada a la corrupción y la incapacidad policial: va a venir una limpieza de fondo

En este caso se atribuye una responsabilidad directa a los elementos de la policía y las instituciones de seguridad como causantes de la inseguridad. El primer supuesto que subyace en el micro-frame de corrupción e ineficiencia policíaca es que las instituciones de seguridad y los elementos policiacos no cumplen sus funciones, por tanto la inseguridad sigue incrementándose. El segundo supuesto que opera en el micro-frame tiene una articulación directa con el macro-frame de enfoque preventivo, en la medida de que la incapacidad policial para atender el problema de la inseguridad está relacionada con el tipo de modelo de seguridad operante.

En el micro-frame de corrupción e incapacidad policial es posible identificar las causas del problema en torno a dos ejes: la corrupción y las competencias. Por un lado, la corrupción se constituye como una de las principales causas de la incapacidad institucional para atender y resolver el problema de la inseguridad. Por otro lado, las deficientes competencias y capacidades de los elementos policiacos parecen imposibilitar el adecuado ejercicio de sus funciones.

La corrupción se presenta como el dispositivo de simplificación del marco más eficiente y significativo utilizado, principalmente, por los candidatos de oposición al partido en el gobierno. El alto grado de resonancia cultural que contiene dicho recurso posibilita que sea utilizado sin necesidad de desarrollar 
ampliamente la relación causal entre la corrupción y la inseguridad: "todos sabemos" que la corrupción es la causa, no solo de la inseguridad sino de la mayoría de los problemas. Tal cualidad permite, también, que se hable de la corrupción de los elementos policiales, de los funcionarios públicos encargados de la seguridad y de cualquier funcionario, político o autoridad. En otras palaras, la corrupción se hace extensiva a toda figura de autoridad, esté o no vinculada directamente con el tema de la seguridad.

Necesitamos una sola visión de seguridad para la zona metropolitana de Guadalajara, pero sobre todo, necesitamos combatir las redes de corrupción que hay detrás del problema de seguridad... (Enrique Alfaro Ramírez, Candidato de MC, Guadalajara, en entrevista con Hechos Noche)

La inseguridad, la violencia, la corrupción y la impunidad es lo que priva y se impulsa día a día... La corrupción es el cáncer que más lacera a la sociedad. (Celia Fausto, candidato del PRD, Guadalajara, debate Académicos UdG)

Las competencias de los elementos de la policía, es decir, sus conocimientos y formación se presentan como un factor importante en el fracaso de las políticas de seguridad. El supuesto es que los policías son responsables por tanto, causantes- de la inseguridad porque no tienen las competencias necesarias para cumplir sus funciones eficiente y eficazmente. Por tanto, predominaron propuestas en materia de capacitación, formación y educación:

Primero un fortalecimiento de las policías con una limpieza de los malos elementos, pero también un respaldo a los buenos elementos policiacos que hay muchos, es decir mejores sueldos, mejor equipamiento, mayor capacitación, mejores seguros de vida en caso de que sucediera alguna cuestión que nadie queramos para ellos, bueno que su familia quede bien protegida. (Pablo Lemus Navarro, Candidato de MC, Zapopan, en entrevista Hechos Meridiano)

Pero la seguridad no solamente es cambiar el modelo, cambiar la capacitación y que tenga mayor equipamiento, se tiene que atender de manera integral. La generación de oportunidades, dignificar la función de los policías... (Salvador Rizo Castelo, Candidato del PRI, Zapopan, en entrevista Meganoticias)

Por supuesto, el incremento de las competencias en los elementos de la policía se plantea acompañado de un conjunto de beneficios sociales y laborales. No se trata, solamente, de "enseñarlos a ser buenos policías", sino también de compensarlos por la labor que desempeñan. 
Hay que armar cuarteles donde podamos tener centros de capacitación y gimnasios para nuestros policías, que tengamos vivienda, con dos objetos, tienes la posibilidad de que estén seguras sus familias y estén seguros nuestros policías... en lugar de estarlos hostigando, simple y sencillamente darles y exigirles la oportunidad de capacitarse y formarse y luego hacer la aplicación del servicio civil de carrera en el ámbito policiaco y darles la oportunidad de crecer... (Guillermo Martínez Mora, Candidato del PAN, Zapopan, Meganoticias)

El micro-frame de corrupción e incapacidad policial está permeado por una importante valoración moral o ética. Ello se evidencia en el recurrente uso de metáforas del tipo buenos policías, malos policías, limpieza de la corporación, limpiar de los malos policías, como podemos observar en los siguientes ejemplos:

...tenemos que mejorar las condiciones para los policías buenos, pero a los policías malos, ya se les acabó también. Va a venir una limpieza de fondo, y es un problema que vamos a ir resolviendo paso a paso... La policía tiene que ser depurada de inicio tenemos que limpiar de los malos policías a la corporación. (Enrique Alfaro Ramírez, Candidato de MC, Guadalajara, en entrevista Hechos Noche)

\section{A modo de conclusión: de los marcos retóricos a la acción pública}

Desde el macro-frame de la prevención las causas de la inseguridad parecen estar clara y plenamente identificadas. Éstas se presentan en torno a tres aristas: la primera relativa a la inoperancia del propio modelo de seguridad; la segunda relacionada con las condiciones estructurales de la desigualdad -el micro-frame de la desigualdad y el de la criminalización de los jóvenes, ilustran cómo opera este enmarcado. La tercera se muestra como un asunto de capacidades institucionales, lo que da sentido a los micro-frames de la corrupción e incapacidad policial y al de la hiper-vigilancia.

De ellas, las causas estructurales de la inseguridad adquieren una mayor preponderancia y énfasis en los discursos de los candidatos. Las causas estructurales se sintetizan en oportunidades laborales, nivel educativo y espacios públicos. El supuesto de fondo es que la desigualdad en el acceso a la educación, al empleo, a las oportunidades de desarrollo, es la causa última de la inseguridad. Estas causas son estructurales, históricas, transversales. Desbordan al municipio y sus márgenes de actuación. Sin embargo, bajo el predominio de este frame, 
todos los candidatos plantearon propuestas que atendieran a ellas. Pero esas propuestas son ambiguas, genéricas, imprecisas: proyectos de educación, más empleos, invertir en educación, políticas educativas, entre otras. En el mejor de los casos se presentaron propuestas nada novedosas, pero sí específicas, que parecen ser posibles desde el ámbito municipal: becas o enseñar algún oficio.

La otra alternativa de solución, consistente con el macro-frame de la prevención, es la policía de proximidad, policía de barrio, policía de cercanía u otras formas en que se denominó a un tipo de elemento de la policía que intenta reestablecer la confianza de la ciudadanía. El policía deberá ser capaz de identificar factores de riesgo y tener la posibilidad de responder con mayor eficiencia a las situaciones de que amenacen la integridad de las personas y sus bienes.

Finalmente, el macro-frame de la prevención se presenta como un marco alternativo a la concepción de la seguridad como el cumplimiento de las normas y la preservación del orden social. En ese sentido, el macro-frame de la prevención enfatiza en atender las causas, los factores, las condiciones que generan o posibilitan la inseguridad o la comisión de delitos. Las condiciones son, básicamente, de tipo estructural (educación, empleo, desigualdad social, etc.), e institucional (capacidades). Por lo tanto, las políticas públicas deben partir de un modelo de seguridad distinto que proponga alternativas de solución en torno a las causas identificadas. Este macro-frame opera en la dimensión retórica de las políticas públicas, en específico en los discursos de los candidatos a un puesto de elección popular. Falta esperar que se materialice en los distintos instrumentos de política pública que deberán orientar la acción pública de los candidatos electos. 


\section{Referencias Bibliográficas}

Bateson, G. (1985). Pasos hacia una ecologia de la mente. Una aproximación revolucionaria a la autocompresión del hombre. Buenos Aires: Ediciones LohléLumen.

Bateson, G. (2006). A Theory of Play and Fantasy. En K. Salen \& E. Zimmerman (Eds.), The Game Design Reader. A Rules of Play Anthology (pp. 314-328). London: The MIT Press.Bryant, J., \& Miron, D. (2004). Theory and Research in Mass Communication. Journal of Communication, 54(4), 662-704.

Bustelo, M., \& Lombardo, E. (2006). Los "marcos interpretativos" de las políticas de igualdad en Europa: conciliación, violencia y desigualdad de género en la política. Revista Española de Ciencia Politica, 14, 117-140.

Castells, M. (2010). Comunicación y poder. España: Alianza Editorial.

Chong, D., \& Druckman, J. N. (2007). Framing Theory. Annual Review of Political Science, 10(1), 103-126.

Entman, R. M. (1993). Framing: Toward Clarification of a Fractured Paradigm. Journal of Communication, 43(4), 51-58.

Epstein, D., Roth, M. C., \& Baumer, E. P. S. (2014). It's the definition, stupid! Framing of online privacy in the Internet Governance Forum debates. Journal of Information Policy, 4, 144-172.

Ferraiolo, K. (2014). Morality Framing in U.S. Drug Control Policy: An Example From Marijuana Decriminalization. World Medical and Health Policy, 6(4), 347374.

Goffman, E. (1986). Frame Analysis. An Essay on the Organization of Experience. Boston: Northeastern University Press.

Goffman, E. (1997). La presentación de la persona en la vida cotidiana. Buenos Aires: Amorrortu Editores.Hulst, M. van, \& Yanow, D. (2014). From Policy "Frames" to "Framing": Theorizing a More Dynamic, Political Approach. American Review of Public Administration, 1, 1-21.

Lima, J. C., \& Siegel, M. (1999). The Tobacco Settlement: An Analysis of Newspaper Coverage of a National Policy Debate, 1997-98. Tabacco Control, 8(3), 247-253.

Luján Cunial, S. (2014). ¿De qué hablan los políticos cuando hablan de drogas? El uso de drogas ilegales como asunto de política pública en Argentina. En V Congreso Uruguayo de Ciencia Politica. Montevideo, Uruguay: Asociación Uruguaya de Ciencia Política.

Magnani, N. (2011). Immigration control in Italian political elite debates: Changing policy frames in Italy, 1998s-2000s. Ethnicities, 12(5), 643-664.

Muñiz, C. (2011). Encuadres noticiosos sobre migración en la prensa digital mexicana. Un análisis de contenido exploratorio desde la teoría del framing. Convergencia, 18(55), 213-239.

Reese, S. D. (2001). Prologue-Framing public life. A bridging model for media research. En S. D. Reese, O. Gandy, \& A. Grant (Eds.), Framing Public Life: Pespectives on Media and our Understanding of the Social World (pp. 7-31). New 
Jersey: Lawrence Erlbaum.Reese, S. D. (2007). The Framing Project: A Bridging Model for Media Research Revisited. Journal of Communication, 57(1), 148-154. Reese, S. D., Gandy, O. H., \& Grant, A. E. (Eds.). (2008). Framing Public Life. Perspectives on Media and Our Understanding of the Social World. New JerseyLondon: Lawrence Erlbaum Associates.

Rein, M., \& Schön, D. (2002). Reframing Policy Discourse. En F. Fischer \& ForesterJohn (Eds.), The Argumentative Turn in Policy Analisys and Planning (pp. 145-166). London: UCL Press.Rose, M., \& Baumgartner, F. R. (2013). Framing the Poor: Media Coverage and U. S. Poverty Policy, 1960 - 2008. Policy Studies Journal, 41(1), 22-53.

Sádaba, T. (2001). Origen, aplicación y límites de la "teoría del encuadre" (framing) en comunicación. Comunicación Y Sociedad, XIV(2), 143-175.

Sádaba, T. (2007). Framing: el encuadre de las noticias. El binomio terrorismomedios. Buenos Aires: La Crujía Ediciones.

Shim, J., Park, C., \& Wilding, M. (2015). Identifying policy frames through semantic network analysis: an examination of nuclear energy policy across six countries. Policy Sci, 48, 51-83.

Tankard, J. W. (2008). The Empirical Aproach to the Study of Media Framing. En S. D. Reese, O. H. Gandy, \& A. E. Grant (Eds.), Framing public life: Perspectives on media and our understanding of the social world (pp. 95-105). New Jersey: Lawrence Erlbaum Associates. Toshkov, D., \& Wieldraaijer, J. (2013). Policy frames and the evolution of soft drugs policy in the Netherlands (1990-2012). En 7th ECPR General Conference (pp. 1-43). Bordeaux: ECPR.

\section{Para citar este artículo:}

Amaya, J., y Portilla, R. (2016). Marcos interpretativos de políticas públicas. El enfoque preventivo de la seguridad en el discurso político de candidatos a presidentes municipales de Guadalajara y Zapopan, Jalisco, México, en las elecciones de 2015. Collectivus, Revista de Ciencias Sociales, 3(1), 36-65. doi: http://dx.doi.org/10.15648/Coll.1.2016.3 